\title{
Severe hypophosphataemia during binge eating in anorexia nervosa
}

\author{
N Kaysar, J Kronenberg, M Polliack, B Gaoni
}

\begin{abstract}
A patient presented with severe hypophosphataemia that had been precipitated during binge eating. It was corrected by restricting the binges, and by hyperalimentation through a duodenal tube together with intravenous supplementation with sodium phosphate for a short period. Phosphate concentrations should be monitored in patients with severe anorexia complicated by bulimic episodes.
\end{abstract}

A low serum phosphate concentration is a complication that has been recorded in several conditions, including hyperalimentation by intravenous fluids deficient in phosphate, ${ }^{12}$ alcohol withdrawal, diabetic ketoacidosis, recovery after extensive burns, and refeeding of malnourished prisoners of war. In anorexic patients hypophosphataemia has been noted during both intravenous hyperalimentation and oral refeeding (when the patients were starved but using laxatives). In some anorexic patients hypophosphataemia may have played a part in the deaths of patients who were treated with intravenous hyperalimentation without phosphate supplementation.

Health Center,

Kupat-Holim,

POB 94,

Hod Hasharon,

Israel 45100

(affiliated to the

Sackler School

of Medicine,

Tel-Aviv University)

N Kaysar

J Kronenberg

M Polliack

B Gaoni

Correspondence to:

Dr Kaysar.

Accepted 28 August 1990

Results of biochemical and haematological investigations

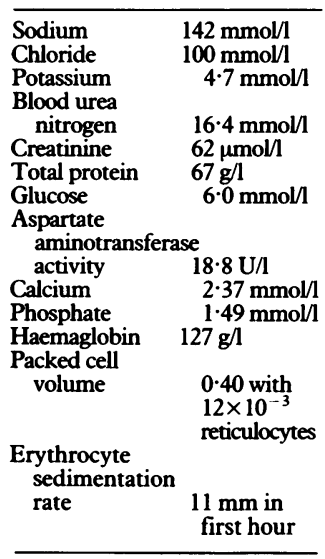

\section{Case report}

A white girl aged 16 years old, $160 \mathrm{~cm}$ in height, had started dieting at the age of 11 years, and was admitted to hospital several times. Her admission to our adolescent's ward (weighing $26 \mathrm{~kg}$ ) was her sixth admission. She had eaten practically nothing except for some unsweetened biscuits, white low fat cheese, and vegetables, and drunk coffee with saccharin. She considered herself fat and wished to weigh $23 \mathrm{~kg}$. She also said that she was using large quantities of laxatives.

On admission she was emaciated and severely cachetic, and complained of feeling cold. Her blood pressure was $80 / 45 \mathrm{~mm} \mathrm{Hg}$ and did not change when she stood up, her pulse rate was $50-64 /$ minute and temperature $36^{\circ} \mathrm{C}$. Her skin was dry and scaly. Initial biochemical values were within the reference range (see table). Urinalysis gave normal results.

During the third week in hospital she started binge eating of large quantities of food from every possible source. Her phosphate concentrations dropped abruptly to $0 \cdot 13,0 \cdot 16$, and $0.26 \mathrm{mmol} / \mathrm{l}$ consecutively. She was placed on a $7.52 \mathrm{MJ}$ diet comprising three meals and three snacks. Her eagerness to binge forced us to restrain her freedom, to feed her through a duodenal tube, and give her sodium phosphate intra- venously. Even after she had been tied to her bed, she persuaded her room mates to bring her food, and even succeeded in pulling out the nasal tube and sucking it directly. Her extreme aggression necessitated antipsychotic drug treatment. She gradually became calmer and we were able to start oral feeding. Her weight was restored and she was discharged from our ward weighing $39 \mathrm{~kg}$.

Follow up consultations took place twice a week. All her biochemical values including phosphorous remain within the reference ranges. Because of her anorexia, however, we are pessimistic about her prognosis.

\section{Discussion}

Phosphorus is essential to the body's energy and storing mechanisms, ${ }^{1-4}$ and it plays a part in carbohydrate, fat, and protein metabolism. In addition, it is necessary for the structure of the phospholipids composing cell membranes and intracellular organelles of muscle and nerve tissue. $^{5}$

Hypophosphataemia can produce the clinical signs of ATP deficiency or cellular hypoxia affecting the neuromuscular, cardiopulmonary, haematological, gastrointestinal, renal, endocrinological, and skeletal systems. ${ }^{67}$ It is a rare clinical entity. The daily requirement is 800 $1000 \mathrm{mg}$ of elemental phosphorus. Dietary sources include eggs, milk, poultry, cheese, nuts, and vegetables. The kidney reabsorbs up to $90 \%$ of phosphorus and stool losses are small, being $100-200 \mathrm{mg} /$ day. ${ }^{3}$ This patient by avoiding practically all food while resorting to laxative abuse reduced her phosphate intake and increased her faecal excretion.

Serum phosphate concentrations reflect only a small proportion of total body phosphorus because $99 \%$ of phosphate is intracellular. ${ }^{8}$ Although within the reference range on admission, her serum phosphate concentration apparently fell precipitously after her binges, thus showing that her total body phosphorus was depleted by her fasting and laxative abuse.

The neurons, red blood cells, and cardiac muscle are particularly vulnerable to ATP depletion. ${ }^{6}$ Presumably the phosphate supplying these tissues from the serum was just adequate during the periods of nutritional deprivation and the catabolic state. The severe bulimic phase changed her body metabolism from a catabolic to an anabolic state, creating an abrupt demand by her liver and muscles for more phosphate for the production of the phosphorylated intermediates required by glycolysis, glycogen formation, and protein synthesis. ${ }^{1}$ 
Phosphate then shifted rapidly from her serum into her muscle and liver. ${ }^{14}$ The drop in serum phosphate threatened to deprive her nervous system, red blood cells, and heart of their phosphate requirement, creating a metabolic emergency.

Hypophosphataemia is a well known cause of death in anorexic patients who are receiving phosphate deficient intravenous hyperalimentation, ${ }^{2}$ and has also been reported in a patient who had been refed orally. ${ }^{1}$ Normal serum concentrations do not exclude the existence of dangerous total body phosphate depletion. We suggest that all binging anorexic patients should have their serum phosphate concentrations monitored and adequate phosphate supplementation should be given. If serum phosphorus concentrations start to drop, the patient should be restrained in bed by all legitimate means to stop her having binges and subsequently misusing laxatives and inducing vomiting.

1 Bheridan PH, Collins $M$. Potentially life-threatening hypophosphataemia in anorexia nervosa. $\mathcal{f}$ Adolesc Health Care 1983;4:44-6.

2 Ruberg RL, Allen TR, Goodman MJ. Hypophosphataemia with hypophosphaturia in hyperalimentation. Surg Forum 1971;22:87-8.

3 Knochel JP. The pathophysiology and clinical characteristics of severe hypophosphatemia. Arch Inter Med 1977;137:203-20.

4 Fitzgerald F. Clinical hypophosphatemia. Annu Rev Med 1978;29:177-89.

5 Staff JP. Phosphate homeostasis and hypophosphatemia. Am $\mathcal{J}$ Med 1982;72:489-95.

6 Waldholtz RA, Andersen AE. Hypophosphatemia during starvation in anorexia nervosa. International fournal of Eating Disorders 1988;7:551-5.

7 Pella B. Medical complication of eating disorders in adolescents. Pediatrics 1988;81:613-23.

8 Lentz RD, Brown DM, Kjellstrand CM. Treatment of severe hypophosphatemia. Ann Intern Med 1978;89:941-4.

9 Bachmund H. Anorexia nervosa, bedropliche neurologische komplikatioren durch hypophosphatamie. Nervenarzt 1986;57:542-4.

Measles and vitamin $A$

Firmly in Michael Caine's 'not many people know that' category is the fact that nearly 60 years ago a paper in the British Medical Fournal pointed to vitamin A having a protective effect in measles. ${ }^{1}$ In 1987 the World Health Organisation recommended vitamin A supplements for children with measles in areas where vitamin A deficiency is rife and where measles has a high mortality. ${ }^{2}$

A recent report from Cape Town (Hussey and Klein, New England Fournal of Medicine 1990;323:160-4) confirms the beneficial effect of vitamin $A$ in severe measles. Altogether 189 children entered a randomised controlled trial receiving either vitamin A $400000 \mathrm{IU}$ or placebo orally. Mortality was $10 \cdot 3 \%$ in the placebo group and $2 \cdot 2 \%$ in the vitamin A group. In the supplemented children the duration of pneumonia was halved and that of diarrhoea reduced by a third. Complications such as post measles croup and herpes stomatitis were significantly reduced.

Measles kills about 2 million children a year so this work is clearly of immense importance especially in developing countries, but low serum concentrations of vitamin $A$ are known to occur during measles even in populations not known to be deficient in the vitamin and the authors therefore advocate giving the supplement to any child who has severe measles.

\section{ARCHIVIST}

1 Ellison JB. Intensive vitamin therapy in measles. BMF 1932;ii:708-11. 1987;62:133-4. 